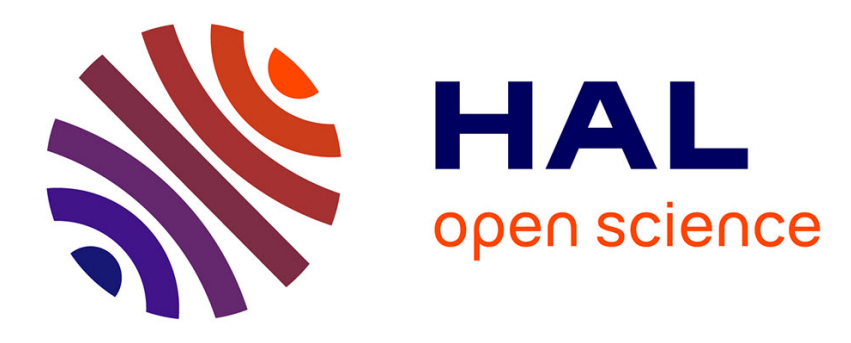

\title{
Protein adsorption mechanisms on solid surfaces: lysozyme-on-mica
}

\author{
Paul Alexander Mulheran, Karina Kubiak
}

\section{To cite this version:}

Paul Alexander Mulheran, Karina Kubiak. Protein adsorption mechanisms on solid surfaces: lysozyme-on-mica. Molecular Simulation, 2009, 35 (07), pp.561-566. 10.1080/08927020802610288 . hal-00515066

\section{HAL Id: hal-00515066 \\ https://hal.science/hal-00515066}

Submitted on 4 Sep 2010

HAL is a multi-disciplinary open access archive for the deposit and dissemination of scientific research documents, whether they are published or not. The documents may come from teaching and research institutions in France or abroad, or from public or private research centers.
L'archive ouverte pluridisciplinaire HAL, est destinée au dépôt et à la diffusion de documents scientifiques de niveau recherche, publiés ou non, émanant des établissements d'enseignement et de recherche français ou étrangers, des laboratoires publics ou privés. 


\section{Molecular Simulation}

Journal of

Experimental Nanoscience

- Taylor \& Francis

Taglor \& Francis Group

\section{Protein adsorption mechanisms on solid surfaces: lysozyme-on-mica}

\begin{tabular}{|r|l|}
\hline Journal: & Molecular Simulation/Journal of Experimental Nanoscience \\
\hline Manuscript ID: & GMOS-2008-0212.R1 \\
\hline Journal: & Molecular Simulation \\
\hline Date Submitted by the \\
Author: & 05-Nov-2008 \\
\hline Complete List of Authors: & $\begin{array}{l}\text { Mulheran, Paul; University of Strathclyde, Chemical and Process } \\
\text { Engineering } \\
\text { Kubiak, Karina; University of Strathclyde, Chemical and Process } \\
\text { Engineering }\end{array}$ \\
\hline Keywords: & $\begin{array}{l}\text { 87.68. Biomaterials and biological interfaces, 87.14. Ee Proteins, } \\
\text { Diffusion of adsorbates, kinetics of coarsening and aggregation } \\
\text { Diflion and }\end{array}$ \\
\hline
\end{tabular}

\section{SCHOLARONE ${ }^{m}$} Manuscripts 
Protein adsorption mechanisms on solid surfaces: lysozyme-on-mica

Paul Mulheran and Karina Kubiak ${ }^{+}$

Department of Chemical and Process Engineering, University of Strathclyde, James Weir Building, 75 Montrose Street, Glasgow G1 1XJ, United Kingdom ${ }^{+}$also at Institute of Physics, Faculty of Physics, Astronomy and Informatics, Nicolaus Copernicus University, ul. Grudziadzka 5/7, 87-100 Torun, Poland

A methodology for discovering the mechanisms and dynamics of protein clustering on solid surfaces is reviewed and complemented by atomistic Molecular Dynamics simulations. Insitu atomic force microscopy images of the early stages of protein film formation are quantitatively compared to Monte Carlo simulations, using cluster statistics to differentiate various growth models. We have studied lysozyme adsorption on mica as a model system, finding that all surface-supported clusters are mobile with diffusion constant inversely related to cluster size. Furthermore, our results suggest that protein monomers diffusing to the surface from solution only adhere to the bare surface with a finite probability. Fully atomistic Molecular Dynamics simulations reveal that the lysozyme does indeed have a preferred orientation for binding to the surface, so that proteins with incorrect orientations move away from the surface rather than towards it. Agreement with experimental studies in the literature for the residues involved in the surface adsorption is found.

\section{Suggested PACS:}

87.68.+z Biomaterials and biological interfaces

87.14.Ee Proteins

87.16.Ac Theory and modeling; computer simulation

68.43.Jk Diffusion of adsorbates, kinetics of coarsening and aggregation 


\section{Mulheran - Protein Adsorption - October 2008 - revision 1}

\section{Introduction}

Protein interaction with solid surfaces is a topic of growing scientific and technological importance. Protein immobilisation at surfaces is desirable for detailed experimental characterisation [1], and necessary for a range of applications such as functionalisation of surfaces and bio-compatible material design $[2,3]$. Current materials science interest centres on bio-mineralisation [4] and other material surface-specific molecular bio-mimetics [5]. The understanding of these processes requires first a sound understanding of how a protein film evolves through the earliest stages of adsorption.

Surprisingly, the mechanisms and dynamics of protein aggregation on solid substrates have, in the past, not been studied in much detail. Random sequential adsorption (RSA) models are widely assumed to be adequate, whereby surface diffusion is at best limited to cluster rearrangements with no long-range centre-of-mass diffusion [6]. However Atomic Force Microscopy (AFM) images repeatedly show clustering of proteins which indicate that such models are inadequate [7-9]. Detailed comparisons between experiment and modelling are rarely found. Furthermore, a methodology to enable a quantitative understanding of both the mechanisms and rates of diffusion and clustering on a solid substrate has, until our recent work, been lacking.

To address this topic, we believe a range of approaches are-is needed, includinge multi-scale modelling. In this paper we review a recently introduced methodology, and supplement this with new, fully atomistic Molecular Dynamics (MD) simulations. Our work shows that progress towards understanding the highly complex adsorption processes and dynamics can be made, although many challenges lie ahead.

\section{Lysozyme adsorption on a mica surface: AFM and Monte Carlo simulations}

A methodology to reveal the mechanisms and dynamics of protein clustering on solid surfaces has recently been presented [10]. The idea is to monitor the evolution of the earliest stages of the protein layer formation using in-situ AFM, comparing the images with suitable Monte Carlo simulations. The simulations are versatile enough to allow several possible 
growth processes to be explored. The statistical properties of the simulations are then characterised in terms of the cluster average size, and the evolution of the cluster size distribution. Comparison of this statistical data with that taken from the AFM then permits the selection of the most successful model, whose dynamics can be quantified from the observed coverage evolution of the surface.

A model system of lysozyme (L6876, Sigma-Aldrich) adsorption onto mica has been used to demonstrate this methodology $[10,11]$. Lysozyme is globular and fairly robust so that reliable observation using in-liquid AFM is viable, and mica provides a flat surface for microscopy whilst presenting a charged surface to attract the protein. Furthermore, lysozyme is small enough to allow atomistic molecular dynamics simulations as described below.

The experiments are performed under stagnant conditions using a very dilute $(1 \mu \mathrm{g} / \mathrm{mL})$ protein solution, so the deposition process lasts many hours allowing adequate time for AFM imaging. A low ionic strength $0.02 \mathrm{M}$ solution (with $\mathrm{pH} 4$ at $22^{\circ} \mathrm{C}$ ) is used to allow electrostatic interactions between the protein and surface, which carry opposite charge at low $\mathrm{pH}$. The experiments show that protein clusters are formed on the surface, and that they are monolayer in height, in agreement with other experimental work [7].

In fig. 1 we present data for the evolution of the fractional surface coverage observed in our experiments. The depth of solution used was $3 \mathrm{~mm}$, allowing us to estimate the final substrate coverage to be 0.36 when the solution is entirely depleted of protein. As can be seen, this final coverage is approached after about 50 hours. The dynamics of the coverage evolution can be modelled by assuming the protein diffuses through the bulk solution and adheres to bare substrate only. With a bulk diffusivity of $D_{b}=1 \times 10^{-6} \mathrm{~cm}^{2} \cdot \mathrm{s}^{-1}[12]$, the coverage is predicted to evolve as the broken line in fig. 1, providing a reasonably good match with the available data. We also investigate the possibility that the protein only sticks to the bare substrate with finite probability $P$; as we shall see below this can be related to the orientation requirements for protein adsorption. The solid curve in fig. 1 is obtained with $P=0.01$, which represents the lower bound for $P$ whilst still providing a satisfactory explanation of the AFM 
data. Note that the effect of this lower value of $P$ is to slow down the evolution of the substrate coverage, thereby providing a higher flux of protein to the surface at later times.

Let us turn now to the statistics of the clusters that self-organise on the surface. In [10] we showed that the distributions cannot be explained using any of the most popular nucleation and growth models. These include Random Sequential Adsorption, where longrange surface diffusion is omitted, and critical island size models where stationary islands nucleate whenever the critical island size $i$ is exceeded on the surface. Instead, a model with mobile clusters is required. We developed a Monte Carlo simulation where monomers (individual proteins) are deposited into a lattice representing the surface at the rate determined from the curves in fig.1. The monomers diffuse by hopping to nearest-neighbour sites; simulations performed with different surface diffusion rates yield different total cluster densities over time. Clusters form when two or more monomers 'collide' by becoming nearest neighbours in the lattice. The clusters themselves can move by rigid translation of their centre-of mass, maintaining their footprints on the lattice. A collision between one cluster and another cluster or monomer results in the formation of a larger cluster. In this way ramified clusters, similar to those observed experimentally $[7,10,11]$, are formed in the simulation.

The cluster mobility $D_{s}$ varies as a power of cluster size (mass) in our simulations $\left(D_{s}=D_{1} s^{-\mu}\right)$. Different values of $\mu$ capture a range of growth mechanisms; $\mu=0$ means all clusters are equally mobile regardless of size, whilst large values of $\mu$ essentially freeze out the movement of all except monomers yielding a critical island size $i=1$ simulation. Furthermore, intermediate values of $\mu$ are expected to reflect the dominant mechanism that causes the cluster mobility: $\mu=0.5$ is expected when monomer evaporation and condensation within a reservoir of surface monomers dominates the cluster mobility; $\mu=1$ when monomer diffusion over/through the cluster dominates; and $\mu=1.5$ when edge diffusion of monomers around clusters dominates. By using the statistics of the simulations to find the best model to describe the experimental data, we identify the dominant growth mechanism. 
In fig.2 we show the cluster size distribution observed in our AFM experiments at the final stages of the protein adsorption from solution [11]. This is compared to results from the simulations that have been found to provide comparable distributions at this and earlier times. With the adsorption probability $P=1$, we find that a low value of $\mu=0.5$ provides a reasonable distribution of cluster sizes, whilst with $P=0.01, \mu=1$ works best. To explain this, we note that at $P=1$ the protein deposition is initially more rapid, so that larger values of $\mu>0.5$ cause the clusters to be effectively immobilised early on, yielding a peaked size distribution reminiscent of a critical island size i>0 model. However with $P=0.01$, the slower deposition means that clusters have more time to explore their neighbourhood before new material arrives, so that $\mu=0.5$ yields a distribution that is overly skewed towards small clusters.

To distinguish the two possible parameter sets, we turn to the evolution of the density of the clusters as shown in fig.3. As can be seen, the $(P=1, \mu=0.5)$ simulations produce a high early peak in cluster density that falls to very low values towards the end of the simulation, a shape that does not compare well with experiment. In contrast, the $(P=0.01$, $\mu=1)$ simulations produce a more realistic density evolution. The final stage of parameterisation is now achieved by adjusting the monomer diffusion rate across the surface, and we find $D_{1} \sim 4.5-9 \times 10^{-16} \mathrm{~cm}^{2} \mathrm{~s}^{-1}[11]$.

To summarise our findings, our simulations indicate two key mechanisms: firstly, not only are the lysozyme monomers mobile on the surface, the clusters they form also appear to diffuse with rates that fall inversely with mass (size); and secondly, the protein only appears to adhere to the bare mica surface with finite probability. In the next section, we show how complementary atomistic simulations can help understand in more detail the second of these conclusions.

\section{Molecular Dynamics simulations}

The crystal structure of Hen Egg-White Lysozyme (HEWL; 1iee.pdb) is the starting structure of all our simulations, with all four disulphide bridges kept. The calculations were 
performed with the CHARMM force field using the NAMD package [13], whilst neutralization, solvation and trajectory visualization were performed using the VMD program [14]. Here we present three different systems. The first comprises the HEWL molecule placed in a rectangular box of water molecules (TIP3) that extends $8 \AA$ from any protein atom, giving a total of over 15000 atoms. Since the net protein charge was $+4 \mathrm{e}$ (at $\mathrm{pH} 7$ ), the system was neutralised by adding $\mathrm{NaCl}$ salt with ionic strength $0.02 \mathrm{M}$. A $20 \mathrm{~ns}$ trajectory of this system provided a reference for trajectories of the protein-surface systems.

Two systems containing the protein molecule and a model mica surface located in different positions with respect to the protein (orientations 1 and 2) have also been simulated. The surface was created from charged silicon $(+1.11 \mathrm{e})$ and oxygen $(-0.66 \mathrm{e})$ atoms, located $1.6 \AA$ away from each other and held rigidly in a square array. The resulting surface charge density $\sigma=-0.0217 \mathrm{e} / \AA^{2}$ is almost equal to that of mica at $\mathrm{pH} 7$ [15]. The neutralized protein was placed close to the surface and then the whole HEWL-surface system was solvated in a water box that extends at least $20 \AA$ from any protein atom and $1 \AA$ from any surface atom. These systems contain $\sim 50000$ atoms, including 1960 protein and 2450 surface atoms.

After initial preparation, all three systems were treated at the same way. First they were subject to $100 \mathrm{ps}$ water equilibration, 10000 steps of whole system minimisation, 30ps heating to $300 \mathrm{~K}$ and $270 \mathrm{ps}$ equilibration at this temperature. The production MD simulations were pursued for $20 \mathrm{~ns}$ at $300 \mathrm{~K}$ in the NVT ensemble. The integration step was $2 \mathrm{fs}$, and the SHAKE algorithm and periodic boundary conditions were used. The cut-off distance for nonbondingthe Coulomb and van der Waals interactions was $12 \AA$. Due to their high computational cost, we have performed short test simulations using the full Ewald summation for the Coulomb interactions. The overall trends are similar for the two approaches, and we only present results for systems with truncated Coulomb interactions. For ionizable residues the most probable charge states at $\mathrm{pH} 7$ were chosen.

Before discussing the surface-protein results, we first consider the isolated protein. In fig. 4 we show the charge distribution across the equilibrated protein surface. It is known that electrostatics play an important role in the adsorption process, since solutions with ionic strength above $0.5 \mathrm{M}$ prevent the adsorption taking place [16]. However there is no obvious positively charge patch on the protein surface that might be expected to interact with the negatively charged mica surface. This highlights the importance of performing molecular modelling which allow structural relaxation in the molecule to occur; models built on the concept of rigid proteins are likely to fail to capture the essence of the process $[15,17]$. 
In fig. 5 we show what happens to the protein over the $20 \mathrm{~ns}$ trajectory, starting from orientation 1. In this orientation, the $\mathrm{C}$ and $\mathrm{N}$ termini of the molecule initially lie close to the surface, with the closest side chain (Arg128) about $8 \AA$ and its backbone about $12 \AA$ away from the surface. At the end of the trajectory, we see that the protein has moved closer to the surface and rotated to allow interaction of Lys1 and Arg128 with the water layers above the surface. The Lys1 and Arg128 side chains have penetrated a water layer and locally disturbed the highly organised hydrogen bond network within the layers. Since the smallest distance between protein side chains and the surface was about $3 \AA$, new chemical bonds have not created. The protein - surface These-interactions also require some flexing of loop regions, but the helical content of the protein is unaffected. The changes in the distance of key residues from the surface are shown in table 1 for clarity. The RMS data (not shown) show that the initial stages of the adsorption process are complete by the end of the $20 \mathrm{~ns}$ trajectory.

The simulation results for the second orientation are shown in fig. 6. In this case, the $\mathrm{C}$ and $\mathrm{N}$ termini of the molecule are oriented away from the surface. The surface is initially located about $6 \AA$ away from the closest HEWL side chain (Arg112) and about $10 \AA$ away from the backbone (Asp48). Over the 20ns trajectory, the protein this time moves away from the surface; see table 2 for the change in the surface separation for reference residues.

These two results indicate that the lysozyme does indeed have a preferred orientation for adsorption on the surface, and helps to justify the inclusion of the finite adsorption probability in the Monte Carlo simulations discussed above. Furthermore there is experimental evidence to support our findings that the most important residues for the interactions between lysozyme and the surface are Arg14, Arg128 and Lys1, all located on the N,C-terminal part of the protein surface. This set is consistent with results obtained by Aizawa et al., who propose that the potential adsorption site is composed from Lys1, Lys13 and Arg 14 [18]. The composition of the main adsorption site found by Dismer et al. [19] also agrees with our selection of residues. Therefore, whilst the 20ns MD trajectories are too short to test for protein unfolding at the surface, it seems that we have correctly identified the early stages of the protein adsorption. This lends support to our modelling of adsorption with a finite probability.

\section{Summary and conclusions}

A new methodology to reveal the mechanisms and dynamics of protein adsorption on solid surfaces has been developed. A combination of AFM imaging and Monte Carlo 


\section{Mulheran - Protein Adsorption - October 2008 - revision 1}

simulation, characterised by appropriate statistics, allows the discovery of the dominant growth mechanisms in the earliest stages of protein layer formation. Applying the methodology to a model system of lysozyme-on-mica, we are led to a (perhaps unexpected) conclusion that protein clusters as well as monomers are mobile on the surface. Furthermore, the simulations indicate how the mobility varies with cluster mass, and allows the quantification of the rates of diffusion. The simulations also require that the protein adheres to the surface with finite probability.

A detailed atomistic understanding of these results is a major challenge. We have made the first steps towards this goal, by studying the initial stages of lysozyme interaction with a model surface using standard Molecular Dynamics simulations. These simulations already support the notion of a finite adsorption probability, since the protein requires a specific orientation to the surface for the adsorption to occur.

A fuller exploration of the interactions with the surface requires much longer timescale simulations. Confrontation with the results for surface diffusion of the protein monomer and clusters remains a daunting task. Nevertheless, the clues provided by the Monte Carlo simulations, namely that the cluster mobility varies inversely with mass, and the diffusion mechanism this implies, provide a focus for our future work.

Acknowledgement: This research is supported by the UK Engineering and Physical Sciences Research Council through grant number EP/E012284. 


\section{References}

[1] R.E. Palmer. C. Leung. 2007. Immobilisation of proteins by atomic clusters on surfaces. Trends in Biotechnology, 25:48.

[2] J.J. Gray. 2004. The interaction of proteins with solid surfaces. Curr. Opin. Struct. Biol. 14:110.

[3] B. Kasemo. 2002. Biological surface science. Surf. Sci. 500:656.

[4] J.H. Harding and D.M. Duffy. 2006. The challenge of biominerals to simulations. J. Mater. Chem. 16:1105.

[5] M. Sarikaya, C. Tamerler, A.K.Y. Jen, K. Schulten, F. Baneyx. 2003. Molecular biomimetics: nanotechnology through biology. Nature Materials 2:577.

[6] J. Talbot, G. Tarjus, P.R. Van Tassel and P. Viot. 2000. From car parking to protein adsorption: an overview of sequential adsorption processes. Colloids Surf. A 165:287.

[7] D.T Kim, H.W. Blanch and C.J. Radke. 2002. Direct imaging of lysozyme adsorption onto mica surface by atomic force microscopy. Langmuir 18:5841.

[8] T.J. McMaster, M.J. Miles, P.R. Shewry and A.S. Tatham. 2000. In situ surface adsorption of the protein C hordein using atomic force microscopy Langmuir 16:1463.

[9] D.C. Cullen and C.R. Lowe. 1994. AFM studies of protein adsorption 1: time-resolved protein adsorption to highly oriented pyrolytic-graphite. J. Colloid Interface Sci. 166:102.

[10] P.A. Mulheran, D. Pellenc, R.A. Bennett, R.J. Green, M. Sperrin. 2008. Mechanisms and dynamics of protein clustering on a solid surface. Phys. Rev. Lett. 100: 068102.

[11] D. Pellenc, R.A. Bennett, R.J. Green, M. Sperrin, P.A. Mulheran. 2008. New insights on growth mechanisms of protein clusters at surfaces: an AFM and simulation study. Langmuir 24: 9648.

[12] S.B. Dubin, A.C. Noel and G.B. Benedek. 1971. Measurement of rotational diffusion coefficient of lysozyme by depolarized light scattering - configuration of lysozyme in solution. J. Chem. Phys. 54:5158. 
[13] J.C. Phillips, R. Braun, W. Wang, J. Gumbart, E. Tajkhorshid, E. Villa, Ch. Chipot, R. D. Skeel, L. Kale, and K. Schulten. 2005. Scalable molecular dynamics with NAMD. J. Comput. Chem. 26:1781.

[14] Humphrey, W., A. Dalke, and K. Schulten. 1996. VMD - Visual Molecular Dynamics. J. Molec. Graphics. 14:33.

[15] F. Carlsson, E. Hyltner, T. Arnebrant, M. Malmsten, and P. Linse. 2004. Lysozyme adsorption to charged surfaces. A Monte Carlo study. J. Phys. Chem. B. 108:9871

[16] T.J. Su, J.R. Lu, R.K. Thomas, Z.F. Cui, and J. Penfold. 1998. The adsorption of lysozyme at the silica -water interface: a neutron reflection study. J. Colloid Interface Sci. 203:419.

[17] S. Ravichandran, J. D. Madura, and J. Talbot. 2001. A Brownian Dynamics study of the initial stages of hen egg-white lysozyme adsorption at a solid interface. J. Phys. Chem. B. 105:3610.

[18] Aizawa, T., N. Koganesawa, A. Kamakura, K. Masaki, A. Matsuura, H. Nagadome, Y. Terada, K. Kawano, and K. Nitta. 1998. Adsorption of human lysozyme onto hydroxyapatite. FEBS Lett. 42:175 - 178.

[19] Dismer, F., M. Petzhold, and J. Hubbuch. 2008. Effects of ionic strength and mobile phase $\mathrm{pH}$ on the binding orientation of lysozyme on different ion-exchange adsorbents. $\mathrm{J}$. Chromat. A. 1194:11 - 21. 


\section{Figure 1}

Coverage of the substrate observed in AFM experiments against exposure time. The smooth curves come from a solution of the diffusion equation, assuming translational symmetry across the system, starting with a uniform distribution of the lysozyme in solution and a bare adsorbing substrate. The sticking coefficient onto bare substrate is $\mathrm{P}=1.0$ and $\mathrm{P}=0.01$.

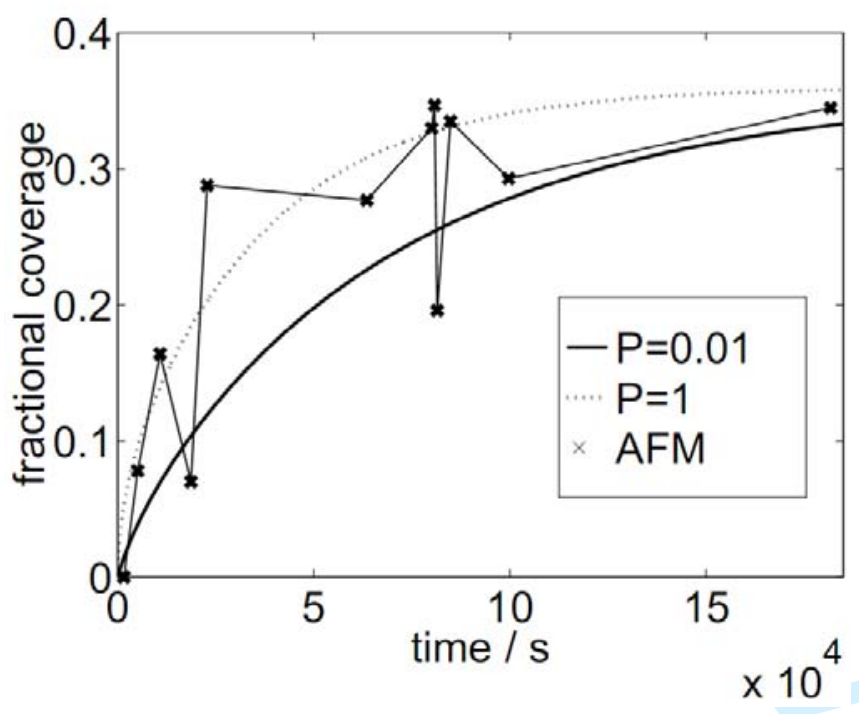




\section{Figure 2}

Cluster size distributions at time 180,000 s. The abscissa is scaled to the mean size. The bars are from the AFM experiments and the lines from the simulations with mobile clusters.

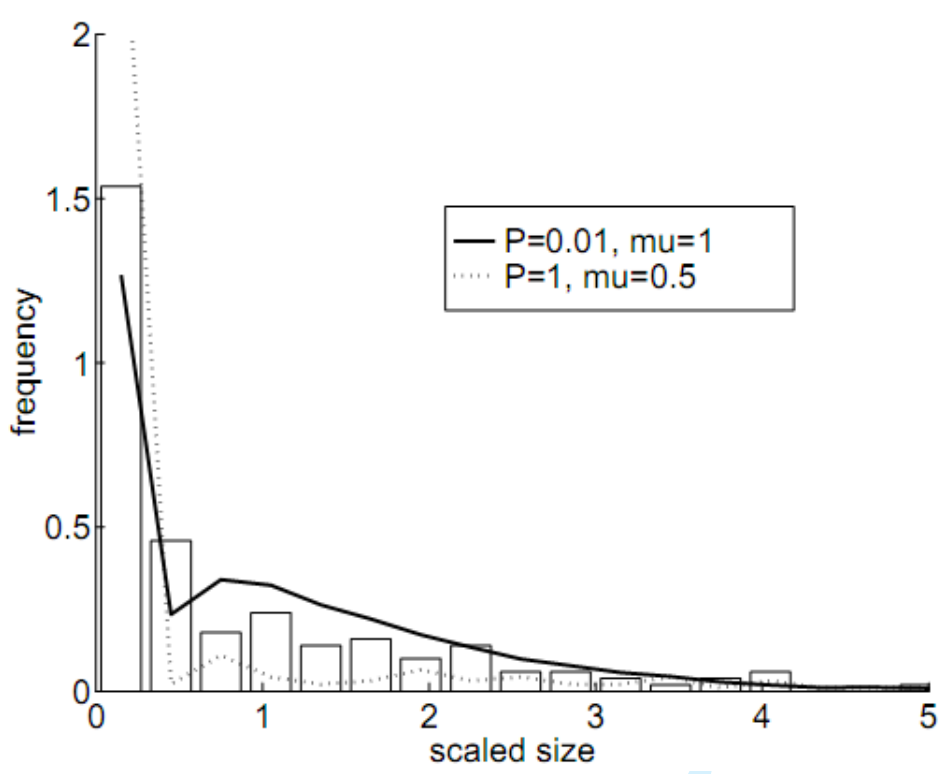




\section{Figure 3}

Cluster density (number per $3 \mathrm{~nm} \times 3 \mathrm{~nm}$ area) evolution over time from AFM images and the simulation models.

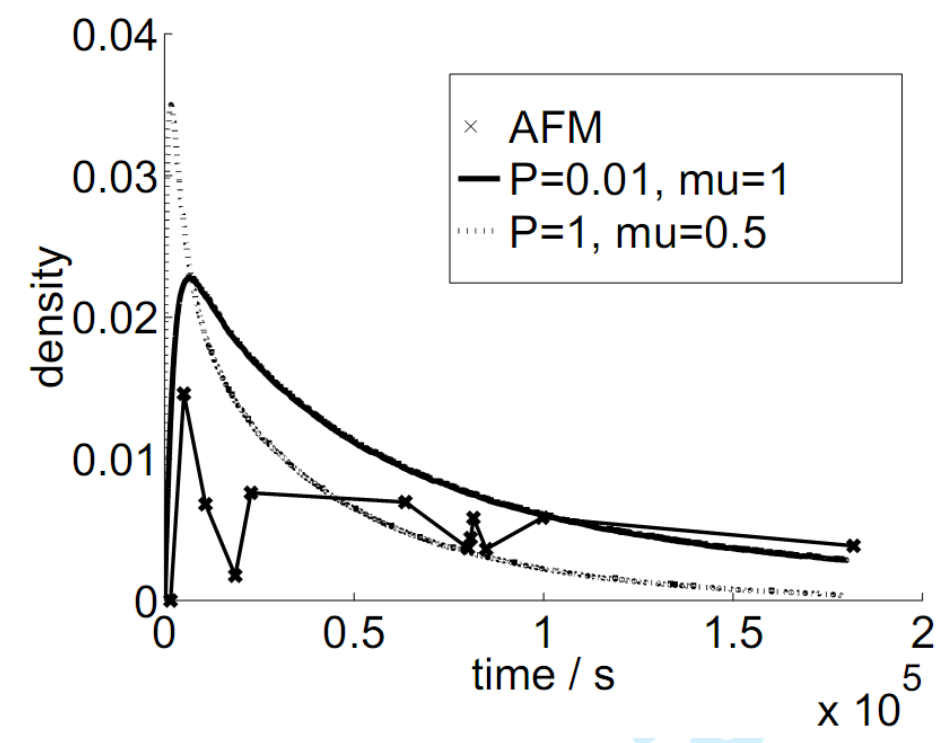




\section{Figure 4 (colour online)}

Surface charge plot of the isolated lysozyme in solution. Red (dark) indicates positive charge, and blue (gray) negative. The protein has a net positive charge of $+4 \mathrm{e}$ at $\mathrm{pH} 7$.

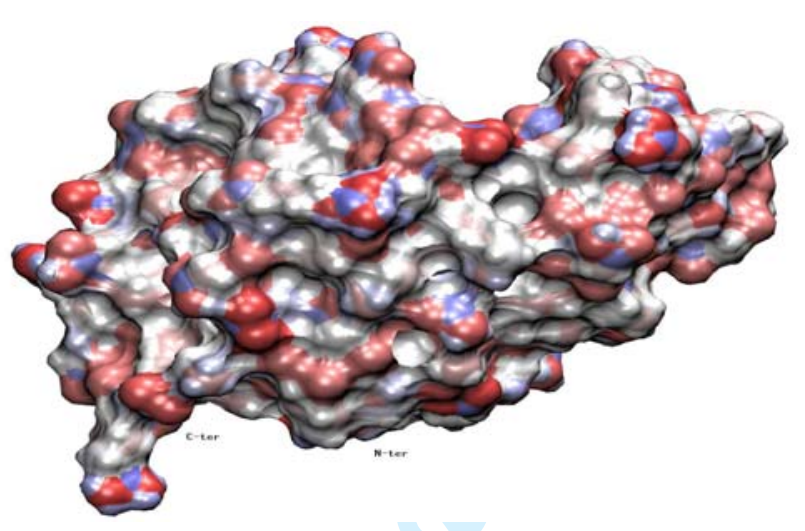




\section{Figure 5 (colour online)}

MD simulation of lysozyme in orientation 1 above the surface. The left image is the initial configuration after thermalisation, and the right is after $20 \mathrm{~ns}$ at $300 \mathrm{~K}$.
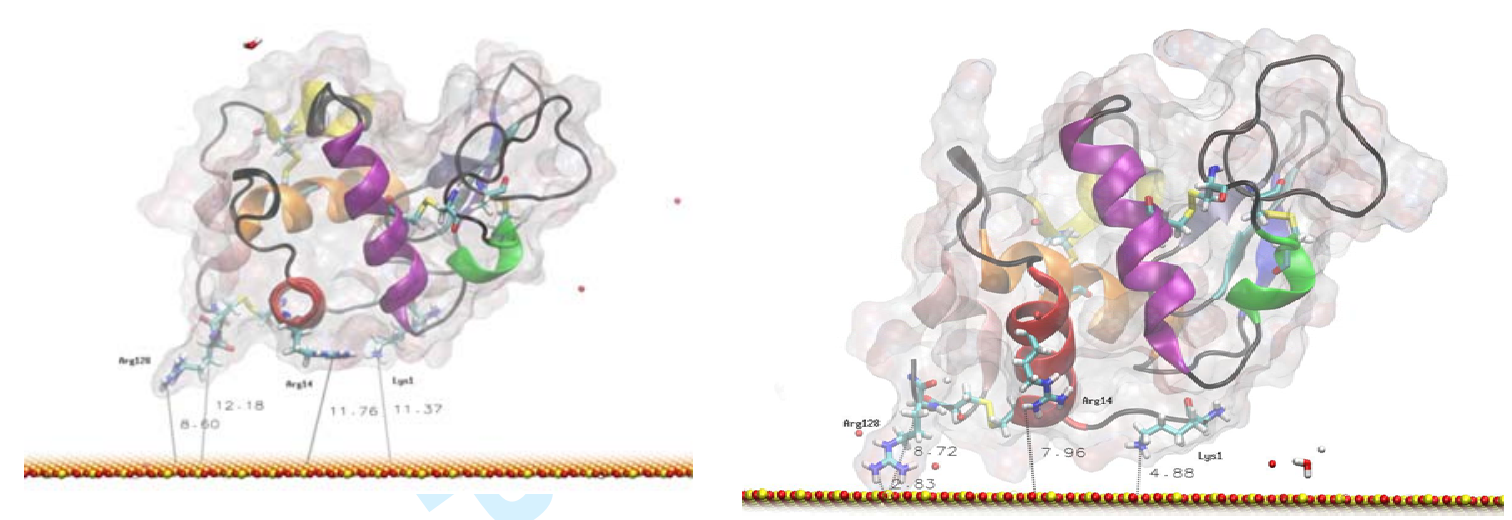


\section{Figure 6 (colour online)}

MD simulation of lysozyme in orientation 2 above the surface. The left image is the initial configuration after thermalisation, and the right is after $20 \mathrm{~ns}$ at $300 \mathrm{~K}$.

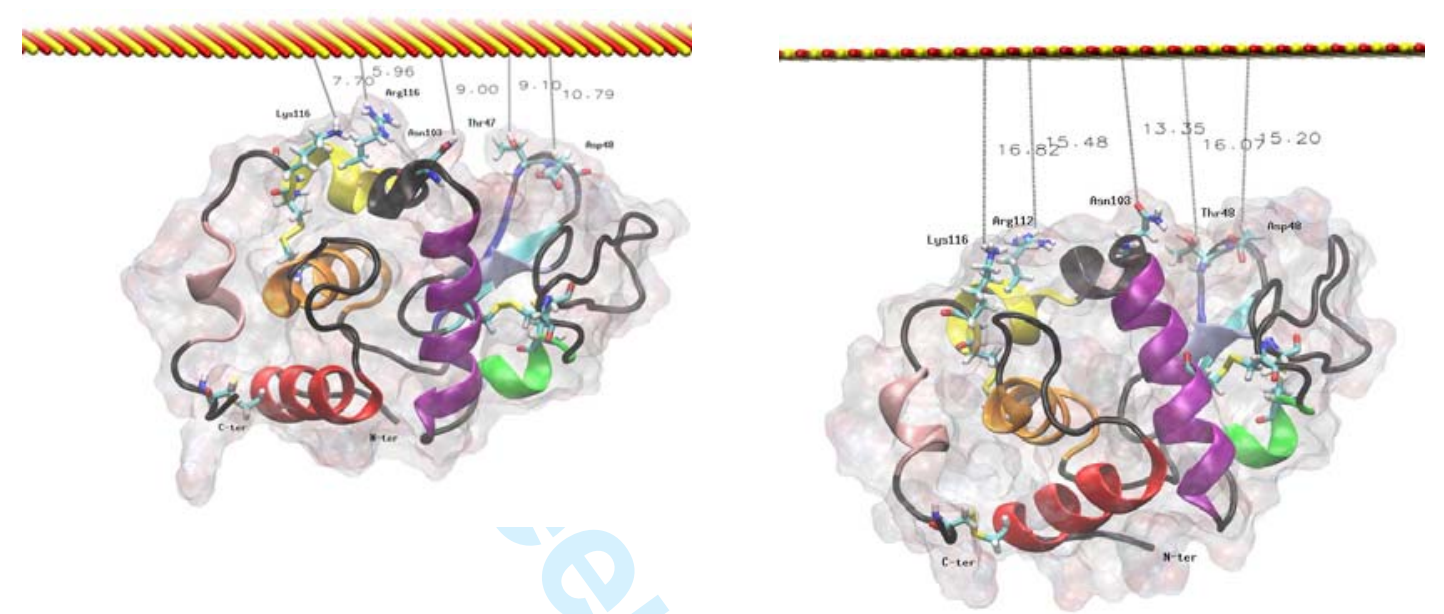




\section{Table 1}

Initial and final distances between the plane of the surface and the key residues involved in the early stages of lysozyme adsorption in orientation 1 (see figure 5).

\begin{tabular}{|llll|}
\hline & \multicolumn{3}{l}{ Distance to the surface $[\AA]$} \\
\cline { 3 - 4 } Residue & Initial & Final & Difference \\
\hline Lys1 & 11.33 & 4.88 & -6.45 \\
Arg14 & 11.76 & 7.96 & -3.80 \\
Arg128 & 8.60 & 2.83 & -5.77 \\
Arg128 & 12.18 & 8.72 & -3.46 \\
\hline
\end{tabular}




\section{Mulheran - Protein Adsorption - October 2008 - revision 1}

Table 2

Initial and final distances between "uppermost" residues in orientation 2 (see figure 6) and the plane of the surface.

\begin{tabular}{|lccc|}
\hline & \multicolumn{3}{l}{ Distance to the surface $[\AA]$} \\
\cline { 2 - 4 } Residue & Initial & Final & Difference \\
\hline Thr47 & 9.91 & 16.07 & +6.16 \\
Asp48 & 10.79 & 15.20 & +4.41 \\
Asn103 & 9.00 & 13.35 & +4.35 \\
Arg112 & 5.92 & 15.48 & +9.56 \\
Lys116 & 7.70 & 16.82 & +9.12 \\
\hline
\end{tabular}

$18 / 18$ 